\title{
Collisional depolarization and transfer rates of spectral lines by atomic hydrogen
}

\section{Application to ionised atoms}

\author{
M. Derouich ${ }^{1}$, S. Sahal-Bréchot ${ }^{1}$, , and P. S. Barklem ${ }^{2}$ \\ 1 Observatoire de Paris-Meudon, LERMA UMR CNRS 8112, 5, Place Jules Janssen, 92195 Meudon Cedex, France \\ 2 Department of Astronomy and Space Physics, Uppsala University, Box 515, 75120 Uppsala, Sweden \\ e-mail: Moncef.Derouich@obspm.fr
}

Received 9 February 2004 / Accepted 29 June 2004

\begin{abstract}
The semi-classical theory of collisional depolarization of spectral lines that we have applied to neutral atoms in previous papers is extended to spectral lines of singly ionised atoms. In order to validate our general theory, we compare our results to quantum chemistry calculations obtained for the particular cases of the $3 \mathrm{~d}^{2} \mathrm{D}$ and $4 \mathrm{p}^{2} \mathrm{P}$ states of the CaII ion. As a demonstration of the universality of our theory and the easiness of its application, we calculate depolarization and polarization transfer rates for the $5 \mathrm{p}^{2} \mathrm{P}$ state of the SrII ion. Analytical expressions of all rates as a function of local temperature are given. Our results for the $\mathrm{CaII}$ ion are compared to recent quantum chemistry calculations. A discussion of our results is presented.
\end{abstract}

Key words. Sun: atmosphere - atomic processes - line: formation - polarization

\section{Introduction}

Observations of the linearly polarized radiation at the limb of the Sun (known as the "second solar spectrum"), which is formed by coherent scattering processes, show rich structures (Stenflo \& Keller 1997; Stenflo et al. 2000; Trujillo Bueno et al. 2001; Bommier \& Molodij 2002). The linear polarization observations reported in the atlas recently published by Gandorfer $(2000,2002)$ show significant polarization peaks in many spectral lines of ions, e.g. NdII $5249 \AA$ A, EuII $4129 \AA$, CeII $4062 \AA$, CeII $4083 \AA$, Ba II D2 $4554 \AA$ A, ZrII $5350 \AA$, etc. Several surveys of the scattering polarization throughout the solar spectrum (Stenflo et al. 1980, 1983a,b; see also the Q/I observations of Stenflo et al. 2000 and the full Stokesvector observations of Dittmann et al. 2001) have shown that ionised lines such as SrII $4078 \AA$ and the IR triplet of CaII are two of the more strongly polarized. The interpretation of these observations requires the solution of the coupling between the polarized radiative transfer equations (RTE) and the statistical equilibrium equations (SEE) taking into account the contributions of isotropic depolarizing collisions with neutral hydrogen. Depolarization and polarization transfer rates are currently available for ionised calcium levels, which have been obtained through sophisticated quantum chemistry methods which are accurate but cumbersome. Indeed, it is very difficult and sometimes not accurate to treat collision processes, involving heavy ionised atoms like Ti II, Ce II, Fe II, Cr II, Ba II..., by standard quantum chemistry methods. It would be useful to develop alternative methods capable of giving results for many levels of ionised atoms rapidly and with reasonable accuracy.

The aim of this paper is to extend the semi-classical theory of collisional depolarization of spectral lines of neutral atoms by atomic hydrogen given in previous papers of this series (Derouich et al. 2003a,b; Derouich et al. 2004; hereafter Papers I, II and III respectively) to spectral lines of ions. This paper outlines the necessary adjustments to the theory presented in Papers I, II and III for extension to spectral lines of ions.

A great advantange of our theory is that it is not specific for a given perturbed ion, and may be easily applied to any singly ionised species. In order to validate this theory, we have compared our results to quantum chemistry calculations when possible. For this purpose, our results are presented and compared with those obtained in the case of CaII levels by the quantum chemistry method (Kerkeni et al. 2003). We also compare the results to the depolarization rates computed with the Van der Waals potential. Indeed, we have applied our method to calculate depolarization and polarization transfer rates for the upper state $5 \mathrm{p}^{2} \mathrm{P}$ of the SrII $4078 \AA$ line.

The main feature of the technique is the use of perturbation theory in calculating the interatomic potentials. A key parameter in this theory is $E_{\mathrm{p}}$ which approximates the energy denominator in the second-order interaction terms by an average value (Paper I and ABO papers: Anstee 1992; Anstee \& O’Mara 1991, 1995; Barklem 1998; Barklem \& O’Mara 1997; 
Barklem et al. 1998). A discussion of the effect of $E_{\mathrm{p}}$ variation on depolarization rates is presented. Finally, we show that the present semi-classical method gives results in agreement with accurate but time consuming quantum chemistry calculations to better than $15 \%$ for the CaII ion $(T=5000 \mathrm{~K})$. Using our method it should now be possible to rapidly obtain the data needed to interpret quantitatively the Stokes parameters of the observed lines.

\section{Statement of the problem}

Under typical conditions of formation of observed lines in the solar atmosphere, the atomic system (atom, ion or molecule) suffers isotropic collisions with hydrogen atoms of the medium before it radiates. The states of the bath of hydrogen atoms are unperturbed. In the tensorial formulation (Fano \& Racah 1959; Messiah 1961; Fano 1963), the internal states of the perturbed particles (here these particles are singly ionised atoms) are described by the spherical tensor components ${ }^{n l J} \rho_{q}^{k}$ of the density matrix. Owing to the isotropy of the depolarizing collisions, the depolarization rates, polarization and population transfer rates are $q$-independent. The term corresponding to the depolarizing collisions in the master equation is given by

$$
\begin{aligned}
\left(\frac{\mathrm{d}{ }^{n l J} \rho_{0}^{k}}{\mathrm{~d} t}\right)_{\text {coll }}= & -\mathrm{D}^{k}(n l J, T){ }^{n l J} \rho_{0}^{k} \\
& -{ }^{n l J} \rho_{0}^{k} \sum_{J^{\prime} \neq J} \zeta\left(n l J \rightarrow n l J^{\prime}, T\right) \\
& +\sum_{J^{\prime} \neq J} \mathrm{D}^{k}\left(n l J^{\prime} \rightarrow n l J, T\right)^{n l J^{\prime}} \rho_{0}^{k} \\
& + \text { quenching term. }
\end{aligned}
$$

$\mathrm{D}^{k}(n l J, T)$ is the collisional depolarization rate of the ionic level $(n l J)$ at the local temperature $T$, where $0 \leq k \leq 2 J$. $\mathrm{D}^{0}(n l J, T)$ is the destruction rate of population which is zero since elastic collisions $\left(J=J^{\prime}\right)$ do not alter the population of the level $(n l J) . \mathrm{D}^{1}(n l J, T)$ is the destruction rate of orientation (related to circular polarization) and $\mathrm{D}^{2}(n l J, T)$ is the destruction rate of alignment of the level $(n l J)$ which is of interest in our astrophysics framework because it is related to the observed linear polarization.

$\zeta\left(n l J \rightarrow n l J^{\prime}, T\right)$ is the fine structure transfer rate between the levels $(n l J) \rightarrow\left(n l J^{\prime}\right)$ and $\mathrm{D}^{k}\left(n l J \rightarrow n l J^{\prime}, T\right)$ is the polarization transfer rate between the levels $|n l J\rangle \rightarrow\left|n l J^{\prime}\right\rangle$, where $0 \leq k \leq k_{\max }, k_{\max }=2 J$ if $J<J^{\prime}$ ( or if $J>J^{\prime}$ then $\left.k_{\max }=2 J^{\prime}\right)$. In particular, $\mathrm{D}^{k}\left(n l J \rightarrow n l J^{\prime}, T\right)$ corresponds to collisional transfer of population $(k=0)$, orientation $(k=1)$ and alignment $(k=2)$.

Higher order terms of $\mathrm{D}^{k}(n l J, T)$ and $\mathrm{D}^{k}\left(n l J \rightarrow n l J^{\prime}, T\right)$ with $k>2$ can play a role in the SEE and have to be calculated. Note that, for the analysis of the linear polarization spectrum, only depolarization and polarization transfer rates with even $k$ are need. Odd $k$-terms can be eliminated from the SEE.

$\mathrm{D}^{k}(n l J, T)$ and $\mathrm{D}^{k}\left(n l J \rightarrow n l J^{\prime}, T\right)$ can be written as a linear combination of the collisional transition rates between the fine stucture sublevels $\zeta\left(n l J M_{J} \rightarrow n l J^{\prime} M_{J}^{\prime}, T\right)$ (Papers I, II and III, Sahal-Bréchot 1977); for depolarization rates $\mathrm{D}^{k}(n l J, T)$ and transfer rate of population

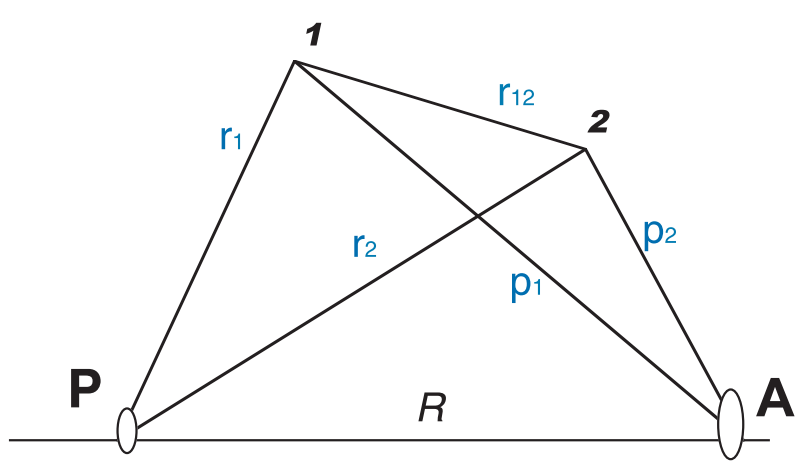

Fig. 1. The perturbed ion core (with charge $Z=2$ ) is located at $A$ and the hydrogen perturbing core (a proton) at $\mathrm{P}$. Their valence electrons are denoted by 1 and 2 respectively.

$\mathrm{D}^{0}\left(n l J \rightarrow n l J^{\prime}, T\right)$, the coefficients of this linear combination are positive while the signs of the coefficients of the linear combination for transfer rates of rank $k \geq 1$ may be either positive or negative. This explains why transfer rates of rank $k \geq 1$ are significantly smaller (Paper III). In our semi-classical theory, the collisional transition rate between the sublevels $\left|n l J M_{J}\right\rangle \rightarrow\left|n l J M_{J}^{\prime}\right\rangle$ is given by (Papers I and II):

$$
\begin{aligned}
\zeta\left(n l J M_{J} \rightarrow n l J M_{J}^{\prime}, T\right)= & n_{\mathrm{H}} \int_{0}^{\infty} \int_{0}^{\infty} 2 \pi b \mathrm{~d} b v f(v) \mathrm{d} v \\
& \times\left|\left\langle n l J M_{J}|I-S(b, v)| n l J M_{J}^{\prime}\right\rangle\right|^{2},
\end{aligned}
$$

where $f(v)$ is the Maxwell distribution of velocities for the local temperature $T$ and $n_{\mathrm{H}}$ is the local hydrogen atom number density. $I$ is the unit matrix and $T=I-S$ is the so-called transition matrix depending on the impact-parameter $\boldsymbol{b}$ and relative velocity $\boldsymbol{v}$. The collisional depolarization rates and the collisional transfer rates, which are linear combinations of the $\zeta\left(n l J M_{J} \rightarrow n l J M_{J}^{\prime}, T\right)$ given by Eq. (2), can be expressed in terms of the $T$-matrix elements. The transition matrix $T$ is functionally dependent on the interaction energy matrix of hydrogen in its ground state with the perturbed ion. Indeed, the transition matrix elements in the dyadic basis are obtained by solving the time-dependent Schrödinger equation (Paper I)

$\left(H_{A}+H_{P}+V_{\text {eff }}(R)\right)|\psi(t)\rangle=\mathrm{i} \frac{\mathrm{d}|\psi(t)\rangle}{\mathrm{d} t}$.

$V_{\text {eff }}$ is the ion-hydrogen interaction used in this work and $|\psi(t)\rangle$ is the wave function of the system (ion+hydrogen). $H_{\mathrm{A}}+H_{\mathrm{P}}$ is the Hamiltonian of the system at the interatomic distance $R=\infty$ (Fig. 1).

\section{Ion-hydrogen interaction potentials}

The interaction potential for a singly ionised atom interacting with a hydrogen atom is treated in much the same way as for the neutral atom interaction with hydrogen (Papers I, II and III; ABO papers). In the coordinate system of Fig. $1, V$ is given, in atomic units, by (Barklem \& O'Mara 1998):

$V=\frac{2}{R}+\frac{1}{r_{12}}-\frac{1}{r_{2}}-\frac{2}{p_{1}}=\frac{1}{R}+\frac{1}{r_{12}}-\frac{1}{r_{2}}-\frac{1}{p_{1}}+V_{\text {ind }}$

and atomic units are used hereafter. $V_{\text {ind }}=1 / R-1 / p_{1}$ is the part representing an inductive interaction between the excess charge 
on the ionised atom and hydrogen atom. Quenching is neglected and thus we consider only the subspace $n l(2 l+1$ states) and we denote the product state of the two separated atoms at $R=\infty$ by $\left|M_{l}\right\rangle$. By application of time-independent perturbation theory to the second order, the interaction potential matrix elements are given by:

$$
\begin{aligned}
\left\langle M_{l}\left|V_{\mathrm{eff}}\right| M_{l}\right\rangle= & \left\langle M_{l}|V| M_{l}\right\rangle \\
& +\sum_{M_{l}^{\prime} \neq M_{l}} \frac{\left\langle M_{l}\left|V_{\mathrm{ind}}\right| M_{l}^{\prime}\right\rangle\left\langle M_{l}^{\prime}\left|V_{\mathrm{ind}}\right| M_{l}\right\rangle}{E_{M_{l}}-E_{M_{l}^{\prime}}} \\
& +\sum_{M_{l}^{\prime} \neq M_{l}} \frac{\left\langle M_{l}\left|V-V_{\text {ind }}\right| M_{l}^{\prime}\right\rangle\left\langle M_{l}^{\prime}\left|V-V_{\text {ind }}\right| M_{l}\right\rangle}{E_{M_{l}}-E_{M_{l}^{\prime}}}
\end{aligned}
$$

$E_{M_{l}}$ are the unperturbed energy eigenvalues of the isolated atoms. The expression for the second-order interaction can be greatly simplified if we replace the energy denominator $E_{M_{l}}-E_{M_{l}^{\prime}}$, of each sum, by a fixed average energy $E_{\mathrm{p}}$ and assume that for important separations $E_{\mathrm{p}}(R)=E_{\mathrm{p}}(\infty)$. This is the Unsöld approximation (Unsöld 1927; Unsöld 1955). $E_{\mathrm{p}}=-4 / 9$ atomic units is the appropriate Unsöld energy value of the part of interaction, $V_{\text {ind }}$, between excess charge on the ionized atom and hydrogen because this part is exactly the same as the $\mathrm{H}-\mathrm{H}^{+}$interaction. Indeed, Unsöld (1927) and Dalgarno \& Lewis (Dalgarno \& Lewis 1956, Eq. (16)) showed that $E_{\mathrm{p}}=-4 / 9$ for the long-range $\mathrm{H}-\mathrm{H}^{+}$interaction. For the part of the interaction describing the interaction between the ion without the excess charge and hydrogen atom, the Unsöld value of $-4 / 9$ cannot be expected to be a good approximation (Barklem \& O'Mara 1998). The reason that a value of $-4 / 9$ works well for neutrals is the fact that the separations of energy levels of the perturbed neutral atom are small compared to the separations between the ground level and the excited levels of the hydrogen atom, and thus the denominators are dominated by contributions from the $\mathrm{H}$ energy levels. For ions this is not the case. As a result of the increased core charge, the energy level spacings are generally much larger than for neutrals. It necessary therefore to determine $E_{\mathrm{p}}$ directly for each state of the ion. The appropriate value of $E_{\mathrm{p}}$ can be found via:

$E_{\mathrm{p}}=-\frac{2\left\langle p_{2}^{2}\right\rangle}{C_{6}}$

where $C_{6}$ is the Van der Waals constant averaged over all $m$ substates. The $C_{6}$ coefficient is given by the standard expression (see for example, Goodisman 1973):

$$
\begin{aligned}
& C_{6}= \\
& \frac{3}{2} \sum_{k^{\prime} \neq k} \sum_{l^{\prime} \neq l} \frac{f_{k k^{\prime}}^{\mathrm{H}} f_{l l^{\prime}}^{\mathrm{A}}}{\left(E_{k^{\prime}}^{H}+E_{l^{\prime}}^{A}-E_{k}^{H}-E_{l}^{A}\right)\left(E_{k^{\prime}}^{H}-E_{k}^{H}\right)\left(E_{l^{\prime}}^{A}-E_{l}^{A}\right)}
\end{aligned}
$$

$f_{l l^{\prime}}^{\mathrm{A}}$ and $f_{k k^{\prime}}^{\mathrm{H}}$ are the dipole oscillator strengths of all transitions to the state of interest $l$ for the perturbed ion and the ground state $k$ for the neutral hydrogen atom. $E^{\mathrm{H}}$ and $E^{\mathrm{A}}$ are the energy eigenvalues of the hydrogen and ionised atom respectively. More details about the calculation of $C_{6}$ are given in Barklem \& O'Mara (1998) and references therein. The quantity $\left\langle p_{2}^{2}\right\rangle$ is the mean square distance between the valence electron and the perturbed ion core located at A (Fig. 1),

$\left\langle p_{2}^{2}\right\rangle=\int_{0}^{+\infty} P_{n^{*} l}^{2}\left(p_{2}\right) p_{2}^{2} \mathrm{~d} p_{2}$,

$P_{n^{*} l}$ are the the radial wavefunctions (note $P_{n^{*} l}\left(p_{2}\right)=R_{n^{*} l} \times$ $\left.\left(p_{2}\right) p_{2}\right)$ of the valence electron of the perturbed atom (Anstee 1992; Seaton 1958). $n^{*}$ is the effective principal quantum number corresponding to the state $|n l\rangle$ of the valence electron (Papers I, II and III).

Using the Unsöld approximation the expression for $V_{\text {eff }}$ becomes

$$
\begin{aligned}
\left\langle M_{l}\left|V_{\text {eff }}\right| M_{l}\right\rangle= & \left\langle M_{l}|V| M_{l}\right\rangle-\frac{1}{E_{\mathrm{p}}}\left(\left\langle M_{l}|V| M_{l}\right\rangle\right)^{2} \\
& +\frac{1}{E_{\mathrm{p}}}\left\langle M_{l}\left|V^{2}\right| M_{l}\right\rangle \\
& +\frac{1}{E_{\mathrm{p}}}\left(\left\langle M_{l}\left|V_{\text {ind }}\right| M_{l}\right\rangle\right)^{2}-\frac{1}{E_{\mathrm{p}}}\left\langle M_{l}\left|V_{\text {ind }}^{2}\right| M_{l}\right\rangle \\
& -\frac{9}{4}\left[\left\langle M_{l}\left|V_{\text {ind }}^{2}\right| M_{l}\right\rangle-\left(\left\langle M_{l}\left|V_{\text {ind }}\right| M_{l}\right\rangle\right)^{2}\right]
\end{aligned}
$$

$V_{\text {eff }}$ of Eq. (9) is the so-called Rayleigh-Schrödinger-Unsöld (RSU) potential. For computing $V_{\text {eff }}$ it is essential to determine $E_{\mathrm{p}}$ in an independent calculation, as seen in Barklem \& O'Mara $(1998,2000)$. Thus for ionized atoms it is not possible to tabulate cross-sections as for neutral atoms (Papers I, II and III). Any calculations for depolarization and transfer of polarization involving ions must proceed line by line.

\section{Determination of depolarization and polarization transfer rates}

Considering a collision between a perturbed ion A and hydrogen atom $\mathrm{H}$ (Fig. 1). Calculation of the depolarization and transfer rates follows essentially the steps listed below:

1. calculation of the required atomic wavefunctions of the system $\mathrm{A}+\mathrm{H}$;

2. determination of $E_{\mathrm{p}}$ directly for each state of the ion using Eq. (6);

3. numerical evaluation of the RSU interaction energy of the system $\mathrm{A}+\mathrm{H}$ given by Eq. (9);

4. use of these interaction potentials in the Schrödinger equation describing the evolution of $\mathrm{A}+\mathrm{H}$ collisional system in order to obtain the probabilities of depolarization and polarization transfer for a given impact parameter and a relative velocity (more details in Paper I; see also Papers II and III);

5. calculation of depolarization and polarization transfer cross-sections for each relative velocity by integration over impact parameters;

6. integration of cross-sections over the Maxwell distribution of velocities to obtain the semi-classical depolarization and polarization transfer rates for a range of local temperatures of the medium. 


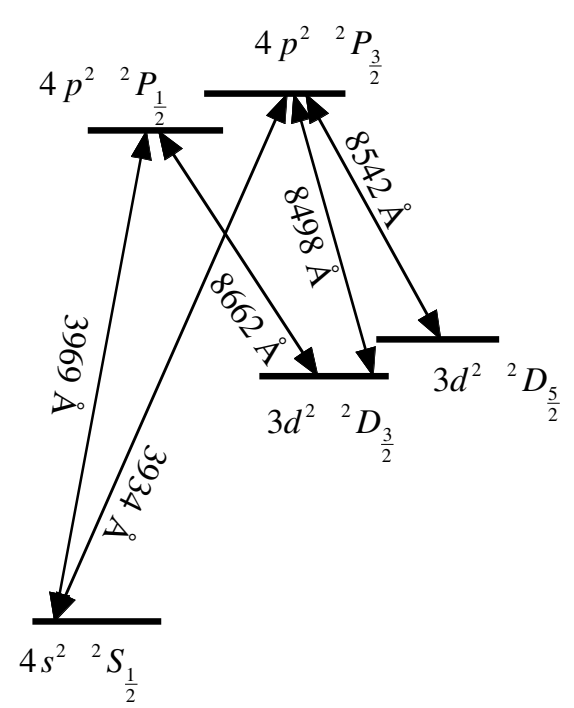

Fig. 2. Partial Grotrian diagram of CaII showing the levels and the spectral wavelengths in $\AA$ of interest in this study. Note that the level spacings in not to scale.

\section{Depolarization and polarization transfer rates for $\mathrm{Ca}^{+}-\mathrm{H}$ system}

An important point to emphasise is that this semi-classical method for the calculation of depolarization and polarization transfer rates is not specific for a given perturbed atom or ion. This method can be applied for any perturbed ion, but we must calculate the $E_{\mathrm{p}}$ value for each case (Sect. 3). Let us consider the case of the $\mathrm{Ca}^{+}-\mathrm{H}$ system in view of its importance in astrophysics and because it is possible to compare with recent calculations employing the quantum chemistry approach (Kerkeni 2003). The case of the IR triplet lines of CaII has been investigated by Manso Sainz \& Trujillo Bueno 2001, 2003 and by Trujillo Bueno \& Manso Sainz 2001, adopting multilevel model of ionized calcium. The term levels associated to the IR triplet lines of CaII (8498 $\AA, 8542 \AA$, and $8662 \AA)$ are $4 \mathrm{p}^{2} \mathrm{P}_{1 / 2}, 4 \mathrm{p}{ }^{2} \mathrm{P}_{3 / 2}, 3 \mathrm{~d}{ }^{2} \mathrm{D}_{3 / 2}$ and $3 \mathrm{~d}^{2} \mathrm{D}_{5 / 2}$ (Fig. 2). The $\mathrm{H}$ and $\mathrm{K}$ lines occur at $3969 \AA$ and $3933 \AA$ respectively (Fig. 2); their upper states are also the upper states of the IR triplet. Table 1 lists, for the states of interest in this work, $\left\langle p_{2}^{2}\right\rangle, C_{6}$ and the corresponding $E_{p}$ calculated via Eq. (6) (see Barklem \& O'Mara 1998).

\subsection{Depolarization rates}

The depolarization transition probability is given by (Paper I; Sahal-Bréchot 1977):

$$
\begin{aligned}
& \left\langle P^{k}(n l J, b, v)\right\rangle_{\mathrm{av}}=\frac{1}{2 J+1} \sum_{\mu, \mu^{\prime}}\left|\left\langle n l J \mu|T| n l J \mu^{\prime}\right\rangle\right|^{2} \\
& -\sum_{\mu, \mu^{\prime}, v, v^{\prime}}\left\langle n l J \mu|T| n l J \mu^{\prime}\right\rangle\left\langle n l J v|T| n l J v^{\prime}\right\rangle^{*} \\
& \quad \times \sum_{\chi}(-1)^{2 J+k+\mu-\mu^{\prime}}\left(\begin{array}{ccc}
J & J & k \\
-v^{\prime} & \mu^{\prime} & \chi
\end{array}\right)\left(\begin{array}{ccc}
J & J & k \\
v & -\mu & -\chi
\end{array}\right) .
\end{aligned}
$$

Table 1. Average energy $E_{\mathrm{p}}$ for the interaction of CaII $3 \mathrm{~d}$ and $4 \mathrm{p}$ states with hydrogen in its ground state together with $\left\langle p_{2}^{2}\right\rangle$ and $C_{6}$ values.

\begin{tabular}{lccc}
\hline \hline State & $\left\langle p_{2}^{2}\right\rangle(\mathrm{au})$ & $C_{6}(\mathrm{au})$ & $E_{\mathrm{p}}(\mathrm{au})$ \\
\hline $3 \mathrm{~d}$ & 7.54 & 12.2 & -1.236 \\
$4 \mathrm{p}$ & 22.25 & 81.8 & -0.544 \\
\hline
\end{tabular}

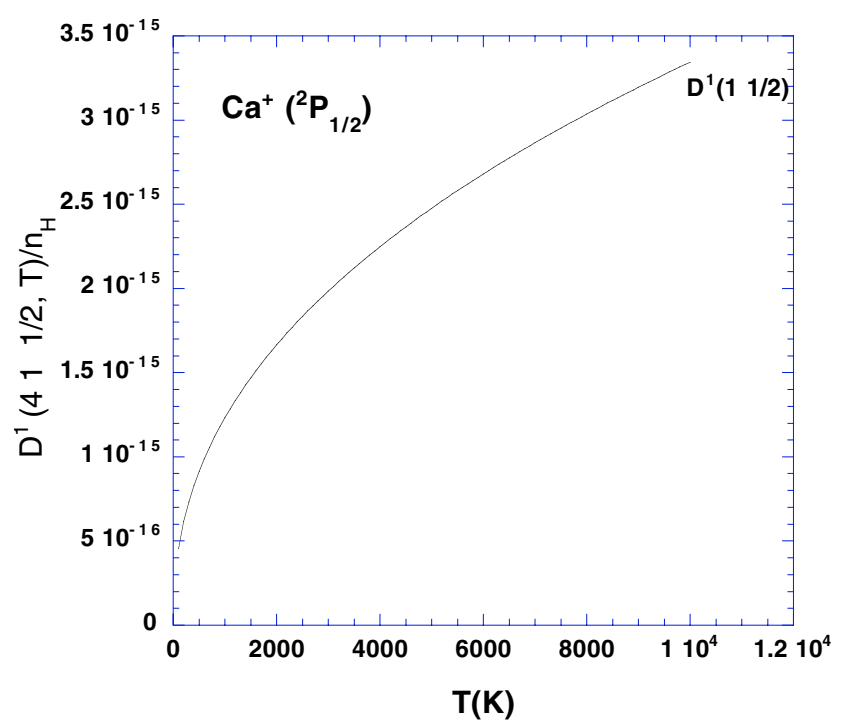

Fig. 3. Destruction rate of orientation per unit H-atom density for the CaII ion, $\mathrm{D}^{1}(411 / 2, T) / n_{\mathrm{H}}$, as a function of temperature $T$. $\mathrm{D}^{1}(411 / 2, T) / n_{\mathrm{H}}$ is given in $\mathrm{rad} \mathrm{m}^{3} \mathrm{~s}^{-1}$.

Owing to the selection rules for the $3 j$-coefficients, the summation over $\chi$ is reduced to a single term, since $\chi=-\left(\mu^{\prime}-v^{\prime}\right)=$ $-(\mu-v)$. Integration over the impact-parameter $b$ and the velocity distribution for a temperature $T$ of the medium can be performed to obtain the depolarization rate which is given by:

$$
\begin{array}{rl}
\mathrm{D}^{k}(n l J, T) \simeq n_{\mathrm{H}} \int_{0}^{\infty} v & f(v) \mathrm{d} v\left(\pi b_{0}^{2}+2 \pi\right. \\
& \left.\int_{b_{0}}^{\infty}\left\langle\mathrm{P}^{k}(n l J, b, v)\right\rangle_{\mathrm{av}} b \mathrm{~d} b\right)
\end{array}
$$

where $b_{0}$ is the cutoff impact-parameter and we use $b_{0}=3 a_{0}$ as in Anstee \& O'Mara (1991). The excited state $4 \mathrm{p}^{2} \mathrm{P}_{1 / 2}$ corresponds to total angular momentum $J=1 / 2$, the only nonzero depolarization rate is $\mathrm{D}^{1}(411 / 2, T)$. Figure 3 shows $\mathrm{D}^{1}(411 / 2, T)$ as a function of the local temperature $T$. The non-zero depolarization rates for the $4 p{ }^{2} \mathrm{P}_{3 / 2}$ state are $\mathrm{D}^{1}(4 \quad 13 / 2, T), \mathrm{D}^{2}(413 / 2, T)$ and $\mathrm{D}^{3}\left(\begin{array}{llll}4 & 1 & 3 / 2, T\end{array}\right)$, and these rates are displayed in Fig. 4. The non-zero depolarization rates associated to the $3 \mathrm{~d}^{2} \mathrm{D}_{3 / 2}$ and $3 \mathrm{~d}^{2} \mathrm{D}_{5 / 2}$ states are $\mathrm{D}^{1}(323 / 2, T), \mathrm{D}^{2}(323 / 2, T), \mathrm{D}^{3}(323 / 2, T)$ for $3 \mathrm{~d}^{2} \mathrm{D}_{3 / 2}$ and $\mathrm{D}^{1}(325 / 2, T), \mathrm{D}^{2}(325 / 2, T), \mathrm{D}^{3}(325 / 2, T)$, $\mathrm{D}^{4}(325 / 2, T)$ and $\mathrm{D}^{5}(325 / 2, T)$ for $3 \mathrm{~d}^{2} \mathrm{D}_{5 / 2}$ (see Figs. 5 and 6).

All of the rates for the $4 p^{2} \mathrm{P}_{1 / 2}, 4 \mathrm{p}^{2} \mathrm{P}_{3 / 2}, 3 \mathrm{~d}^{2} \mathrm{D}_{3 / 2}$ and $3 \mathrm{~d}$ ${ }^{2} \mathrm{D}_{5 / 2}$ states of CaII are found to increase with temperature in 


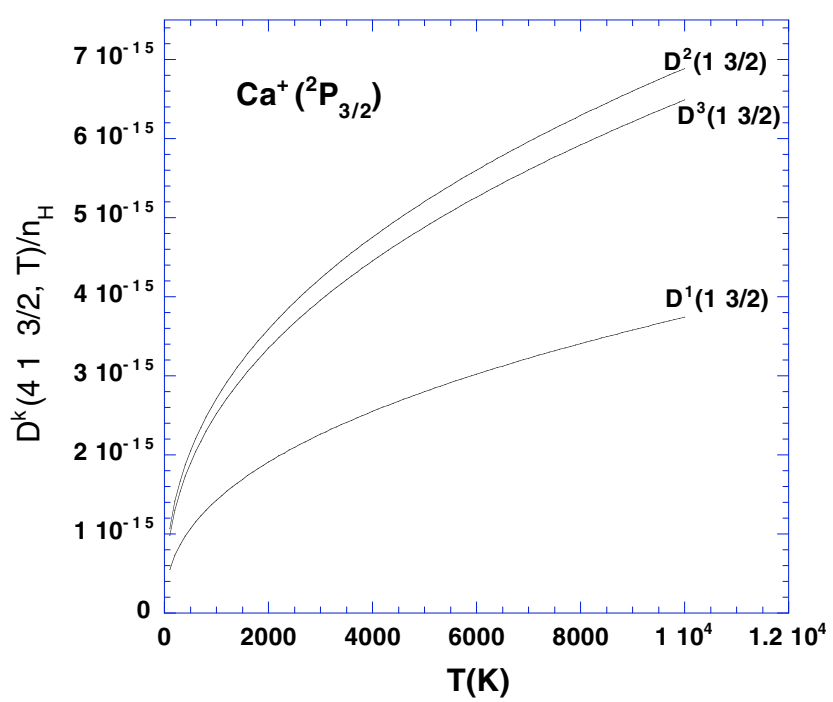

Fig. 4. Depolarization rates per unit $\mathrm{H}$-atom density for the CaII ion, $\mathrm{D}^{k}(413 / 2, T) / n_{\mathrm{H}}(k=1,2$, and 3$)$, as a function of temperature $T$. $\mathrm{D}^{k}(413 / 2, T) / n_{\mathrm{H}}$ are given in $\mathrm{rad} \mathrm{m}^{3} \mathrm{~s}^{-1}$.

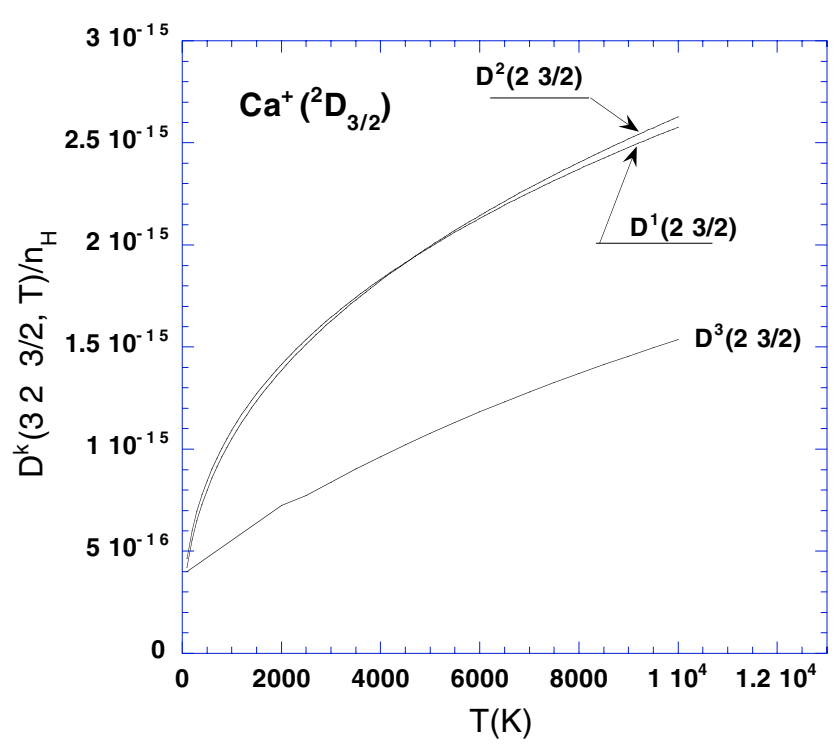

Fig. 5. Depolarization rates per unit $\mathrm{H}$-atom density for the CaII ion, $\mathrm{D}^{k}(323 / 2, T) / n_{\mathrm{H}}(k=1,2$, and 3$)$, as a function of temperature $T$. $\mathrm{D}^{k}(323 / 2, T) / n_{\mathrm{H}}$ are given in $\operatorname{rad~m}^{3} \mathrm{~s}^{-1}$.

the range $100 \leq T \leq 10000 \mathrm{~K}$. As for neutral atoms, a functional form $\mathrm{D}(T)=B T^{(1-\lambda) / 2}$ can usually be accurately fitted to these depolarization rates, where $\lambda$ is the so-called velocity exponent (Papers I, II and III). We find the following analytical expressions for the depolarization rates for $100 \leq T \leq$ $10000 \mathrm{~K}$ (except for $\mathrm{D}^{3}(325 / 2, T)$ and $\mathrm{D}^{5}(325 / 2, T)$ which are given for $2500 \leq T \leq 10000 \mathrm{~K}$ ):

- CaII $\left(4 \mathrm{p}^{2} \mathrm{P}_{1 / 2}\right)-\mathrm{H}(1 \mathrm{~s})$ :

$$
\begin{aligned}
\mathrm{D}^{1}(411 / 2, T)= & 2.4767 \times 10^{-15} \\
\times & \times n_{\mathrm{H}}\left(\frac{T}{5000}\right)^{0.433}\left(\mathrm{rad} \mathrm{m}^{3} \mathrm{~s}^{-1}\right)
\end{aligned}
$$

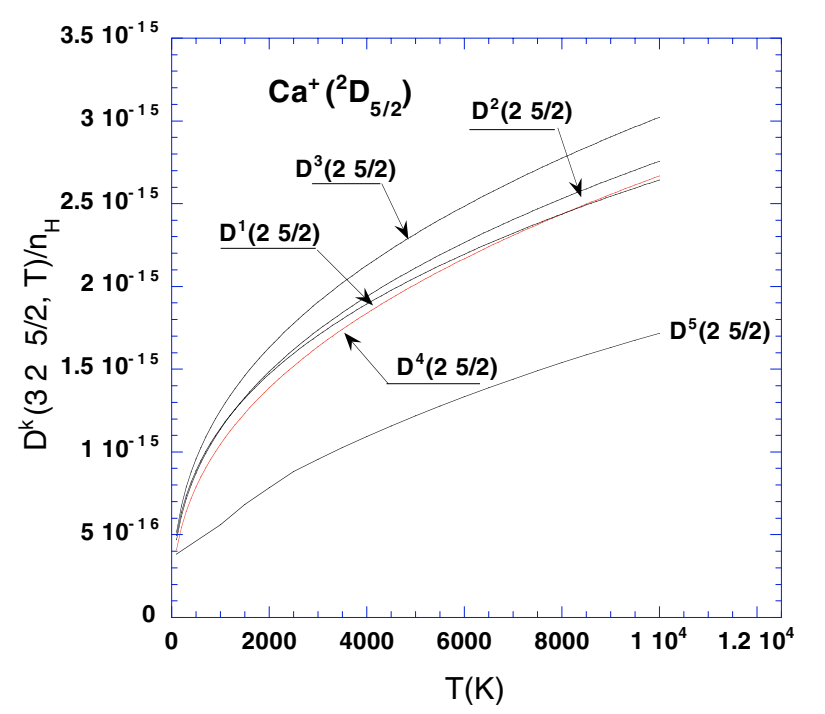

Fig. 6. Depolarization rates per unit $\mathrm{H}$-atom density for the $\mathrm{CaII}$ ion, $\mathrm{D}^{k}(325 / 2, T) / n_{\mathrm{H}}(k=1,2,3,4$, and 5), as a function of temperature $T$. $\mathrm{D}^{k}(325 / 2, T) / n_{\mathrm{H}}$ are given in $\mathrm{rad} \mathrm{m}^{3} \mathrm{~s}^{-1}$.

$$
\begin{aligned}
&-\mathrm{CaII}\left(4 \mathrm{p}^{2} \mathrm{P}_{3 / 2}\right)-\mathrm{H}(1 \mathrm{~s}): \\
& \mathrm{D}^{1}(413 / 2, T)= 2.7993 \times 10^{-15} \\
& \times n_{\mathrm{H}}\left(\frac{T}{5000}\right)^{0.418}\left(\mathrm{rad} \mathrm{m}^{3} \mathrm{~s}^{-1}\right) \\
& \mathrm{D}^{2}(413 / 2, T)= 5.2034 \times 10^{-15} \\
& \times n_{\mathrm{H}}\left(\frac{T}{5000}\right)^{0.405}\left(\mathrm{rad} \mathrm{m}^{3} \mathrm{~s}^{-1}\right)
\end{aligned}
$$

$\mathrm{D}^{3}(413 / 2, T)=4.8807 \times 10^{-15}$

$$
\times n_{\mathrm{H}}\left(\frac{T}{5000}\right)^{0.411}\left(\mathrm{rad} \mathrm{m}^{3} \mathrm{~s}^{-1}\right)
$$

- $\operatorname{CaII}\left(3 \mathrm{~d}^{2} \mathrm{D}_{3 / 2}\right)-\mathrm{H}(1 \mathrm{~s})$ :

$\mathrm{D}^{1}(323 / 2, T)=1.9904 \times 10^{-15}$

$$
\times n_{\mathrm{H}}\left(\frac{T}{5000}\right)^{0.373}\left(\mathrm{rad} \mathrm{m}^{3} \mathrm{~s}^{-1}\right)
$$

$\mathrm{D}^{2}(323 / 2, T)=1.9943 \times 10^{-15}$

$$
\times n_{\mathrm{H}}\left(\frac{T}{5000}\right)^{0.398}\left(\mathrm{rad} \mathrm{m}^{3} \mathrm{~s}^{-1}\right)
$$

$\mathrm{D}^{3}(323 / 2, T)=1.0772 \times 10^{-15}$

$$
\times n_{\mathrm{H}}\left(\frac{T}{5000}\right)^{0.501}\left(\mathrm{rad} \mathrm{m}^{3} \mathrm{~s}^{-1}\right),
$$

- CaII $\left(3 \mathrm{~d}^{2} \mathrm{D}_{5 / 2}\right)-\mathrm{H}(1 \mathrm{~s})$ :

$\mathrm{D}^{1}(325 / 2, T)=2.0535 \times 10^{-15}$

$$
\times n_{\mathrm{H}}\left(\frac{T}{5000}\right)^{0.365}\left(\mathrm{rad} \mathrm{m}^{3} \mathrm{~s}^{-1}\right)
$$

$\mathrm{D}^{2}(325 / 2, T)=2.1120 \times 10^{-15}$

$$
\times n_{\mathrm{H}}\left(\frac{T}{5000}\right)^{0.384}\left(\mathrm{rad} \mathrm{m}^{3} \mathrm{~s}^{-1}\right)
$$

$\mathrm{D}^{3}(325 / 2, T)=2.3170 \times 10^{-15}$

$$
\times n_{\mathrm{H}}\left(\frac{T}{5000}\right)^{0.384}\left(\mathrm{rad} \mathrm{m}^{3} \mathrm{~s}^{-1}\right)
$$


$\mathrm{D}^{4}(325 / 2, T)=2.0127 \times 10^{-15}$

$$
\begin{aligned}
& \times n_{\mathrm{H}}\left(\frac{T}{5000}\right)^{0.407}\left(\mathrm{rad} \mathrm{m}^{3} \mathrm{~s}^{-1}\right) \\
\mathrm{D}^{5}(325 / 2, T)= & 1.2187 \times 10^{-15} \\
& \times n_{\mathrm{H}}\left(\frac{T}{5000}\right)^{0.486}\left(\mathrm{rad} \mathrm{m}^{3} \mathrm{~s}^{-1}\right) .
\end{aligned}
$$

\subsection{Polarization transfer rates}

The collisional transfer transition probability is given by (Paper II; Sahal-Bréchot 1977):

$$
\begin{aligned}
& \left\langle P^{k}\left(n l J \rightarrow n l J^{\prime}, b, v\right)\right\rangle_{\mathrm{av}}= \\
& \sum_{\mu, \mu^{\prime}, v, v^{\prime}}\left\langle n l J \mu|T| n l J^{\prime} \mu^{\prime}\right\rangle\left\langle n l J v|T| n l J^{\prime} v^{\prime}\right\rangle^{*}
\end{aligned}
$$

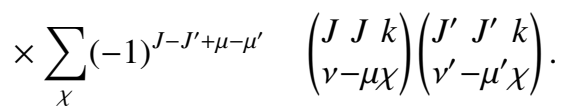

As in Eq. (11), the polarization transfer rates $\mathrm{D}^{k}(n l J \rightarrow$ $\left.n l J^{\prime}, T\right)$ follow from integration over the impact parameters and the velocities with a Maxwellian distribution.

Inelastic collisions with neutral hydrogen which leave the radiating atom in a final state $n^{\prime} l^{\prime}$ different from the initial one $n l$ are neglected. Only the polarization transfer rates inside the subspace $n l$ are taken into account. Our transfer rates between the levels $4 \mathrm{p}^{2} \mathrm{P}_{1 / 2} \rightarrow 4 \mathrm{p}^{2} \mathrm{P}_{3 / 2}, 3 \mathrm{~d}^{2} \mathrm{D}_{3 / 2} \rightarrow 3 \mathrm{~d}^{2} \mathrm{D}_{5 / 2}$ $\left(\mathrm{D}^{k}(411 / 2 \rightarrow 413 / 2, T)\right.$ and $\left.\mathrm{D}^{k} 1(411 / 2 \rightarrow 413 / 2, T)\right)$ are presented in Figs. 7 and 8 respectively. $\mathrm{D}^{3}\left(\begin{array}{llll}3 & 2 & 3 / 2 & \rightarrow\end{array}\right.$ $325 / 2, T)$ did not obey a power law of the form $B T^{(1-\lambda) / 2}$. However, we can provide the analytical expressions for the other non-zero transfer rates:

$$
\begin{aligned}
\mathrm{D}^{0}(411 / 2 \rightarrow 413 / 2, T)= & 4.0307 \times 10^{-15} \\
& \times n_{\mathrm{H}}\left(\frac{T}{5000}\right)^{0.407}\left(\mathrm{rad} \mathrm{m}^{3} \mathrm{~s}^{-1}\right)
\end{aligned}
$$

$\mathrm{D}^{1}(411 / 2 \rightarrow 413 / 2, T)=-1.1464 \times 10^{-15}$

$$
\times n_{\mathrm{H}}\left(\frac{T}{5000}\right)^{0.314}\left(\mathrm{rad} \mathrm{m}^{3} \mathrm{~s}^{-1}\right)
$$

$\mathrm{D}^{0}(323 / 2 \rightarrow 325 / 2, T)=1.8061 \times 10^{-15}$

$$
\times n_{\mathrm{H}}\left(\frac{T}{5000}\right)^{0.392}\left(\mathrm{rad} \mathrm{m}^{3} \mathrm{~s}^{-1}\right)
$$

$\mathrm{D}^{1}(323 / 2 \rightarrow 325 / 2, T)=1.6177 \times 10^{-16}$

$$
\begin{aligned}
& \times n_{\mathrm{H}}\left(\frac{T}{5000}\right)^{1.401}\left(\mathrm{rad} \mathrm{m}^{3} \mathrm{~s}^{-1}\right) \\
\mathrm{D}^{2}(323 / 2 \rightarrow 325 / 2, T)= & 8.6286 \times 10^{-16} \\
& \times n_{\mathrm{H}}\left(\frac{T}{5000}\right)^{0.490}\left(\mathrm{rad} \mathrm{m}^{3} \mathrm{~s}^{-1}\right) .
\end{aligned}
$$

\section{Comparisons}

It is important to notice that the depolarization rates $\mathrm{D}^{k}(n l J, T)$ as defined in this work (Eq. (11)) and the relaxation rates $g^{k}(J)$ as defined by Kerkeni et al. (2003) (Eq. (2), Sect. 3.2) are not equivalent. Kerkeni et al. (2003) defines the

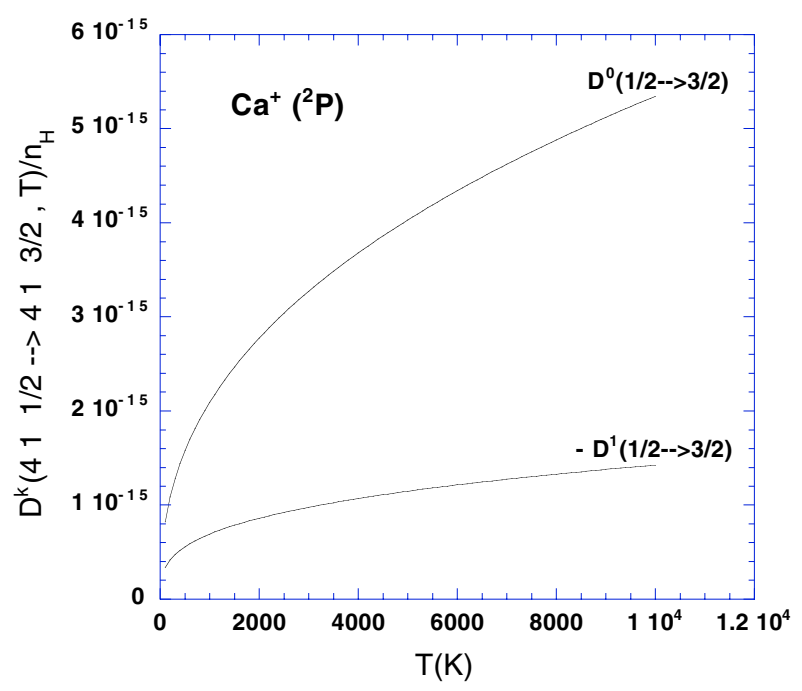

Fig. 7. Population and orientation transfer rates $(k=0$ and $k=1)$, per unit $\mathrm{H}$-atom density, as a function of temperature $T$. The rates are given in $\mathrm{rad} \mathrm{m}^{3} \mathrm{~s}^{-1}$.

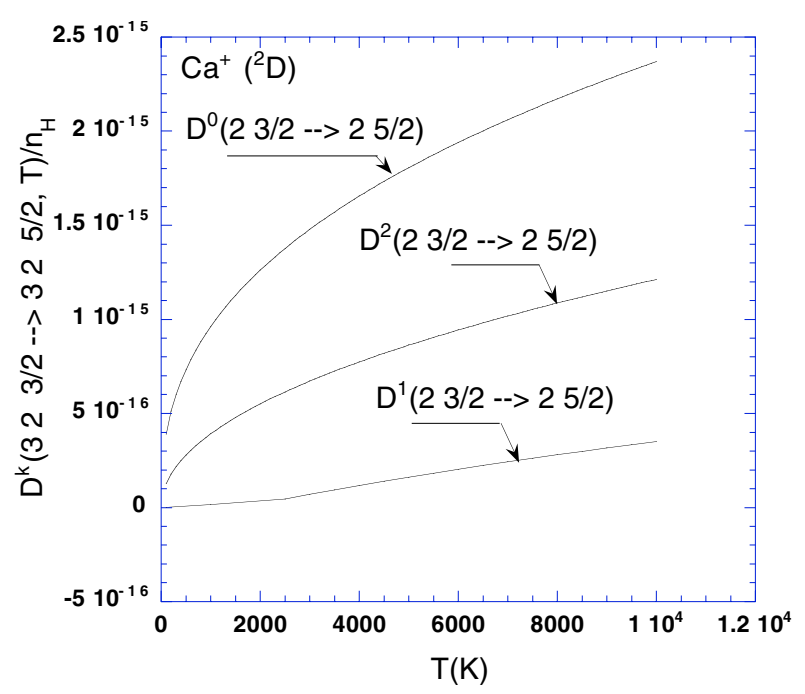

Fig. 8. Polarization transfer rates per unit $\mathrm{H}$-atom density, $\mathrm{D}^{k}(323 / 2 \rightarrow 325 / 2, T) / n_{\mathrm{H}}$, as a function of temperature $T$. The rates are given in $\mathrm{rad} \mathrm{m}^{3} \mathrm{~s}^{-1}$.

depolarization cross-section (or relaxation rate $g^{k}(J)$ ) as the sum of two terms: the term responsible exclusively for the depolarization of the level $(n l J)$ and the term corresponding to the fine structure transfer between the levels $(n l J) \rightarrow\left(n l J^{\prime}\right)$. In our definition, $\mathrm{D}^{k}(n l J, T)$ is only the depolarization of the level $(n l J)$, the fine structure transfer is not included. We calculate separately the rates $\zeta\left(n l J \rightarrow n l J^{\prime}, T\right)$ associated to fine structure transfer which are $k$-independent and proportional to the population transfer rates $\mathrm{D}^{0}\left(n l J \rightarrow n l J^{\prime}, T\right)$ (Eq. (4) of Paper II).

For example, in accordance with our definition $\mathrm{D}^{0}(n l l, \quad T) \equiv 0$ but the relaxation rates $g^{0}(J)$ from Kerkeni et al. (2003) are not necessarily zero. In order to compare to the Kerkeni et al. (2003) results it is essential to substract the part of $g^{k}(J)$ associated to fine structure transfer $\left(g\left(J \rightarrow J^{\prime}\right)\right.$ in Kerkeni et al. 2003). This it is nothing more 

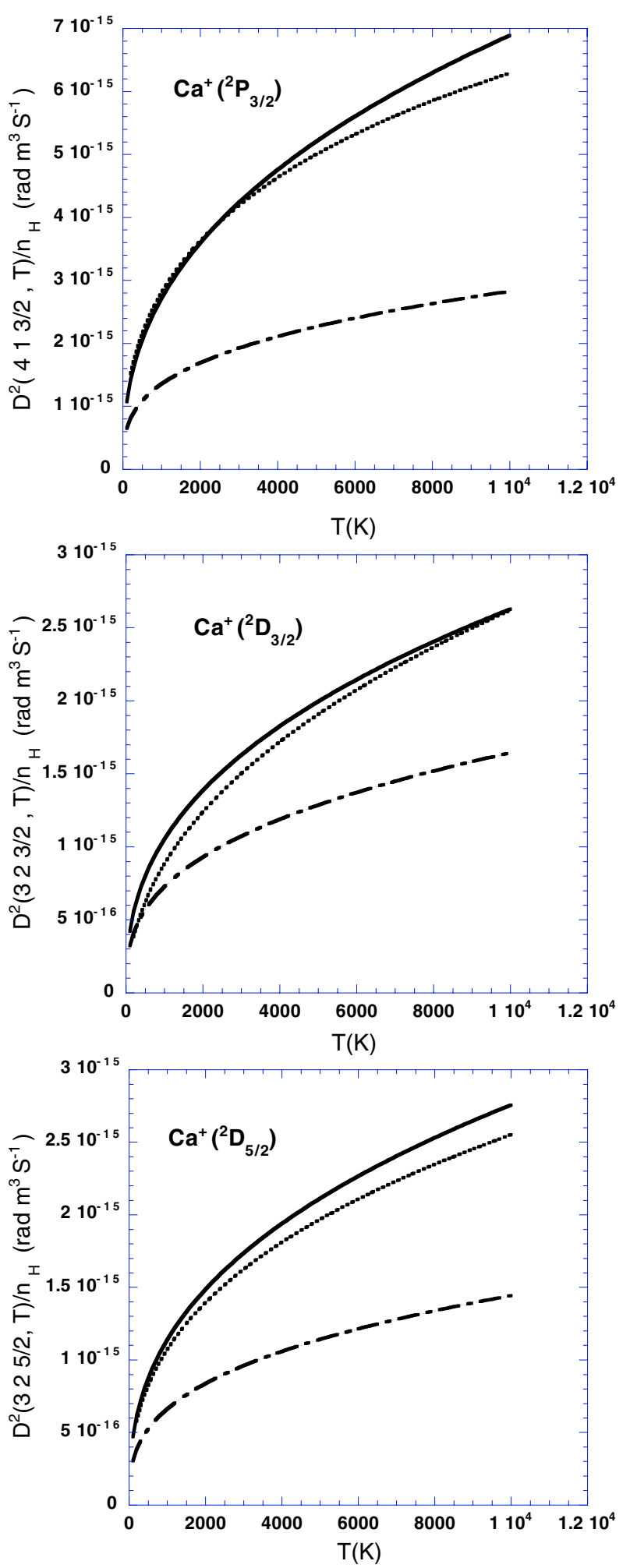

Fig. 9. Depolarization rates for $k=2$ as a function of temperature. Full lines: our results; dotted lines: quantum chemistry calculations (Kerkeni 2003); dot-dashed lines Van der Waals approximation.

than a difference in definitions. Nevertheless, this difference should be taken into account when writing the SEE. We now compare our alignment depolarization rates with the quantum chemistry depolarization rates (Kerkeni et al. 2003) and the alignment depolarization rates obtained by replacing the RSU

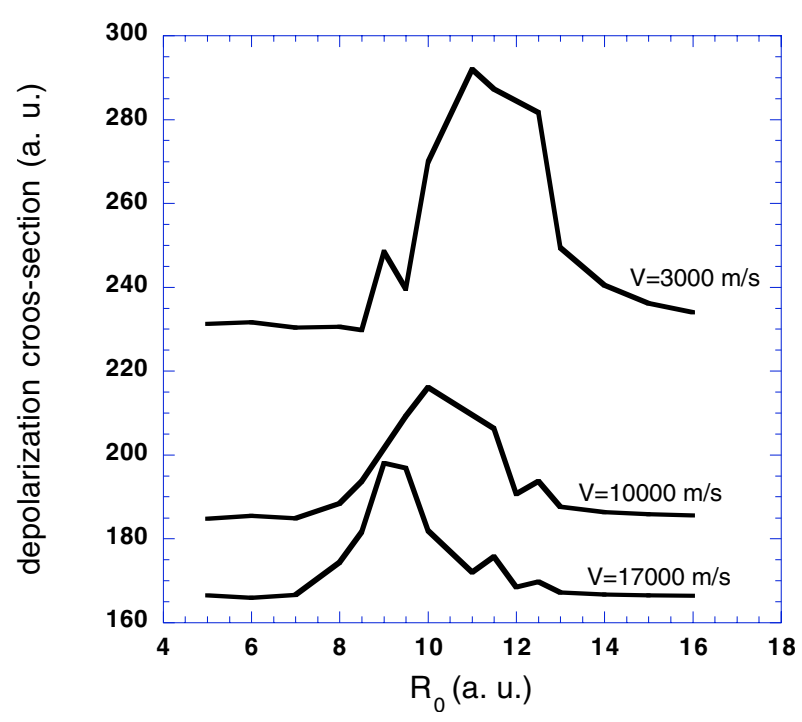

Fig. 10. Depolarization cross section enhancement due to a Gaussian local perturbation of the potential. Cross sections are calculated for the relative velocities: $3 \mathrm{~km} \mathrm{~s}^{-1}, 10 \mathrm{~km} \mathrm{~s}^{-1}$ and $17 \mathrm{~km} \mathrm{~s}^{-1}$.

potential with improved Van der Waals potential, $V=-\frac{C_{6}}{R^{6}}$, where $C_{6}$ is taken from Table 1 . Note that the latter rates differ from the usual Van der Waals formula for the rates, because though we employ the van der Waals potential, the collision dynamics are treated using our theory, and the $C_{6}$ value is accurately determined (whereas typically the approximation $C_{6}=\alpha_{\mathrm{H}}\left\langle p_{2}^{2}\right\rangle$ is employed where $\alpha_{\mathrm{H}}$ is the polarisability of hydrogen). In Fig. 9 we show our alignment depolarization rates with quantum chemistry depolarization rates (Kerkeni et al. 2003) and the improved Van der Waals rates. We display only the $k=2$ case which is related to the linear depolarization (alignment). Reference to Fig. 9 shows that the Van der Waals potential significantly underestimates the depolarization cross section. Our results show rather good agreement with quantum chemistry calculations. In concrete terms, the percentage errors at $T=5000 \mathrm{~K}$ with respect to quantum chemistry depolarization rates are $4.1 \%$ for $\mathrm{D}^{2}(413 / 2, T) / n_{\mathrm{H}} ; 4.6 \%$

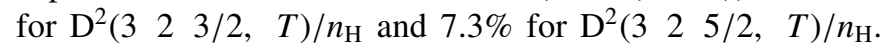
The errors for the other depolarization and transfer rates are similar to the errors for the destruction rates of alignment (in general, less than 15\%). This similarity is expected since all rates orginate from the same collisional processes.

\section{Discussion}

\subsection{Dependence of depolarization cross-sections on interatomic separations}

To assess the sensitivity of the calculations to the accuracy of the potentials at various separations we make calculations with potentials where we have introduced local perturbations. The interaction potential $(1 \mathrm{~s}, 4 \mathrm{p} \sigma)$ is multiplied by a Gaussian magnification factor of the form (Anstee \& O'Mara 1991):

$G(R)=1+\exp \left(-\left(R-R_{0}\right)^{2}\right)$.

$R_{0}$ is the position where the variation of $V_{\mathrm{eff}}$ reaches its maximum value (the interaction potential ( $1 \mathrm{~s}, 4 \mathrm{p} \sigma$ ) doubles). 


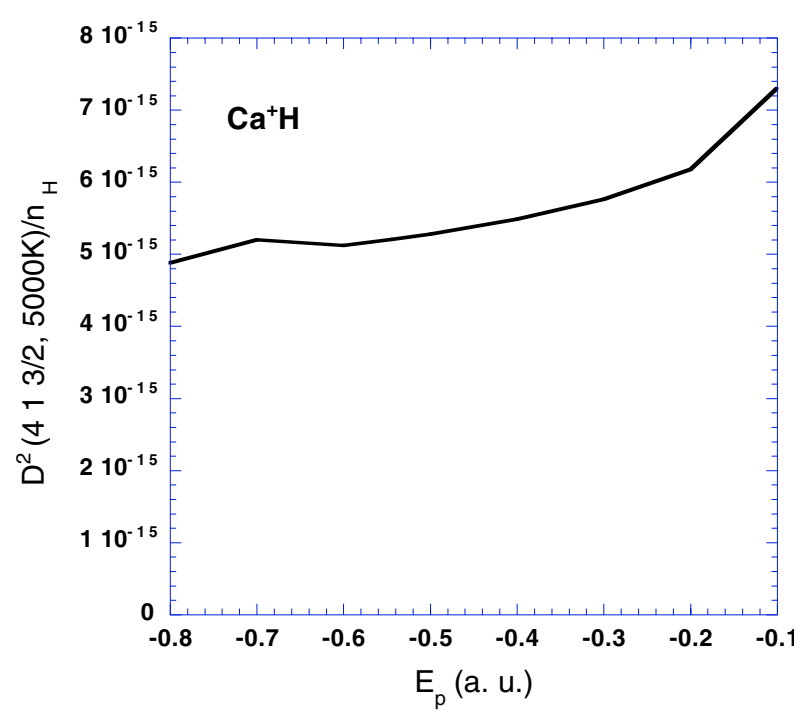

Fig. 11. Dependence on $E_{\mathrm{p}}$ of the destruction rate of alignment $\mathrm{D}^{2}(413 / 2,5000 \mathrm{~K}) / n_{\mathrm{H}}$.

Figure 10 shows the depolarization cross-section calculated with varying $R_{0}$. It is clear that the values of $R_{0}$ inducing depolarization cross-section enhancement confirm the fundamental result found already for neutral atoms: the interactions of importance for the depolarization cross-section (or depolarization rate) calculations are the intermediate-range interactions (Papers I, II and III). The principal differences between the RSU potentials and those from quantum chemistry occur at small interatomic separations. It is for this reason that we obtain rather good agreement with quantum chemistry calculations. The Van der Waals interaction potentials are inaccurate at the intermediate region, and this explains why these potentials underestimate the depolarization cross sections.

\subsection{Dependence of depolarization rates on $E_{p}$}

The depolarization and transfer rates for the $4 p$ and $3 d$ states are calculated for $E_{\mathrm{p}}=-0.544$ and -1.236 respectively. As a check on the sensivity of our results to the precision of the calculations of $E_{\mathrm{p}}$, we have calculated the destruction rate of alignment $\mathrm{D}^{2}(413 / 2,5000 \mathrm{~K})$ by varying $E_{\mathrm{p}}$ in Eq. (9). Note that when $E_{\mathrm{p}}$ decreases, the interaction potential decreases and so $\mathrm{D}^{2}(413 / 2,5000 \mathrm{~K}$ ) also decreases (Fig. 11). The depolarization rate shows only an extremly weak variation with $E_{\mathrm{p}}$. An $\left|E_{\mathrm{p}}\right|$ variation of $25 \%$, with respect to the value $E_{\mathrm{p}}=-0.544$, corresponds to a change of less than $5 \%$ in the calculated depolarization rates. It should not concluded that this is a general property of the depolarization rates for all states of all ionised atoms. Reference to Fig. 11 shows a rather strong dependence of the depolarization rates on $E_{\mathrm{p}}$ for $\left|E_{\mathrm{p}}\right| \leq 0.25$. We expect, however, that the value of $\left|E_{\mathrm{p}}\right|$ is usually greater than 0.25 and the depolarization rates will not be greatly affected by possible error in the value of $E_{\mathrm{p}}$ (see Barklem \& O'Mara 1998, 2000).

\section{Application to the Sr II $4078 \AA$ line}

The Sr II $4078 \AA$ line was examined by Bianda et al. (1998), who wrote "...The rather large uncertainty in the depolarizing collision rate introduces a corresponding uncertainty in the field-strength scale...". These authors have used the traditional Van der Waals approach to calculate collisional rates.

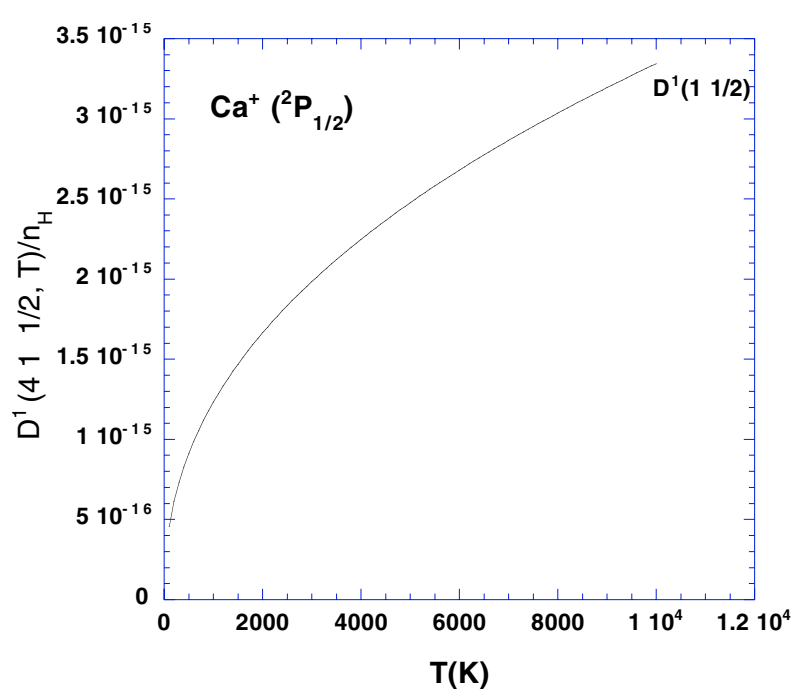

Fig. 12. Destruction rate of orientation per unit $\mathrm{H}$-atom density for the SrII ion, $\mathrm{D}^{1}(511 / 2, T) / n_{\mathrm{H}}$, as a function of the temperature of the medium, $T$. $\mathrm{D}^{1}(511 / 2, T) / n_{\mathrm{H}}$ is given in $\operatorname{rad~}^{3} \mathrm{~s}^{-1}$.

The $4078 \AA$ line is the resonance line of SrII: $5 \mathrm{~s}{ }^{2} \mathrm{~S} \rightarrow 5 \mathrm{p}{ }^{2} \mathrm{P}$. We have computed the depolarization and polarization transfer rates of the levels $5 p^{2} \mathrm{P}_{1 / 2}$ and $5 \mathrm{p}^{2} \mathrm{P}_{3 / 2}$ of SrII ion. The value of $E_{\mathrm{p}}=-0.564$ for the level $5 \mathrm{p}^{2} \mathrm{P}$ of SrII was adopted from Barklem \& O'Mara (2000). We applied our method to obtaining the rates which are shown in Figs. 12 and 13. They were found to again obey a power law $A T^{(1-\lambda) / 2}$ and are given for $100 \leq T \leq 10000 \mathrm{~K}$ by:

$$
\begin{aligned}
&-\operatorname{SrII}\left(5 \mathrm{p}^{2} \mathrm{P}_{1 / 2}\right)-\mathrm{H}(1 \mathrm{~s}): \\
& \mathrm{D}^{1}(511 / 2, T)= 2.7196 \times 10^{-15} \\
& \times \times n_{\mathrm{H}}\left(\frac{T}{5000}\right)^{0.428}\left(\mathrm{rad} \mathrm{m}^{3} \mathrm{~s}^{-1}\right)
\end{aligned}
$$

- $\operatorname{SrII}\left(5 \mathrm{p}^{2} \mathrm{P}_{3 / 2}\right)-\mathrm{H}(1 \mathrm{~s})$ :

$$
\begin{aligned}
\mathrm{D}^{1}(513 / 2, T)= & 3.1560 \times 10^{-15} \\
& \times n_{\mathrm{H}}\left(\frac{T}{5000}\right)^{0.418}\left(\mathrm{rad} \mathrm{m}^{3} \mathrm{~s}^{-1}\right)
\end{aligned}
$$$$
\mathrm{D}^{2}(513 / 2, T)=5.9776 \times 10^{-15}
$$

$$
\times n_{\mathrm{H}}\left(\frac{T}{5000}\right)^{0.406}\left(\mathrm{rad} \mathrm{m}^{3} \mathrm{~s}^{-1}\right)
$$

$\mathrm{D}^{3}(513 / 2, T)=5.5413 \times 10^{-15}$

$$
\times n_{\mathrm{H}}\left(\frac{T}{5000}\right)^{0.410}\left(\mathrm{rad} \mathrm{m}^{3} \mathrm{~s}^{-1}\right) .
$$

Between the term levels $5 \mathrm{p}{ }^{2} \mathrm{P}_{1 / 2}$ and $5 \mathrm{p}{ }^{2} \mathrm{P}_{3 / 2}$ there are only two non-zero polarization transfer rates which are given in Fig. 14. The analytical expressions for these rates for $100 \leq$ $T \leq 10000 \mathrm{~K}$ are:

$$
\begin{aligned}
\mathrm{D}^{0}(511 / 2 \rightarrow 513 / 2, T)= & 4.6184 \times 10^{-15} \\
& \times n_{\mathrm{H}}\left(\frac{T}{5000}\right)^{0.409}\left(\mathrm{rad} \mathrm{m}^{3} \mathrm{~s}^{-1}\right)
\end{aligned}
$$

$\mathrm{D}^{1}(511 / 2 \rightarrow 513 / 2, T)=-1.5309 \times 10^{-15}$

$$
\times n_{\mathrm{H}}\left(\frac{T}{5000}\right)^{0.338}\left(\operatorname{rad~m}^{3} \mathrm{~s}^{-1}\right) .
$$




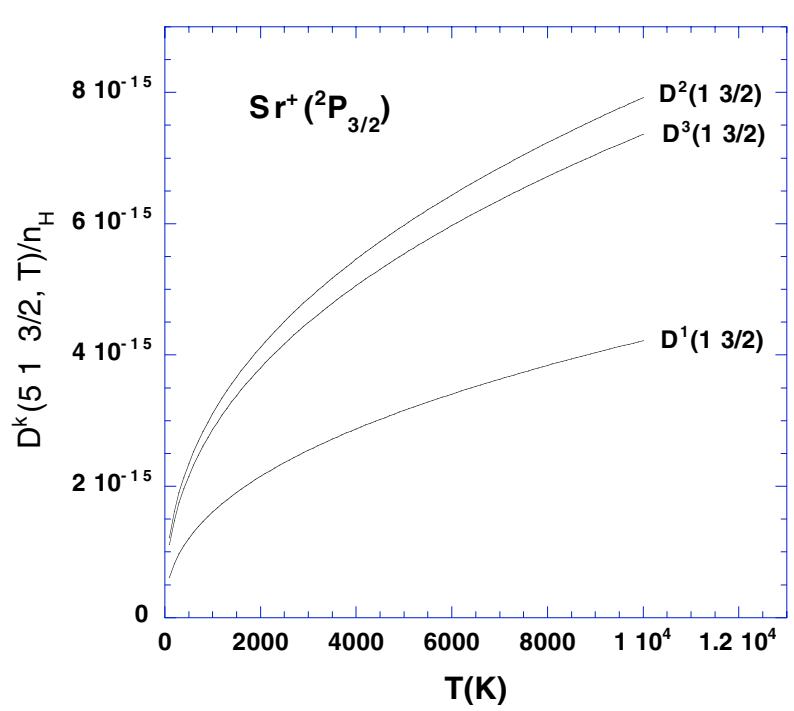

Fig. 13. Depolarization rates, $\mathrm{D}^{k}(513 / 2, T) / n_{\mathrm{H}}(k=1,2$, and 3$)$, as a function of temperature $T$. $\mathrm{D}^{k}(513 / 2, T) / n_{\mathrm{H}}$ are given in $\operatorname{rad~m}^{3} \mathrm{~s}^{-1}$.

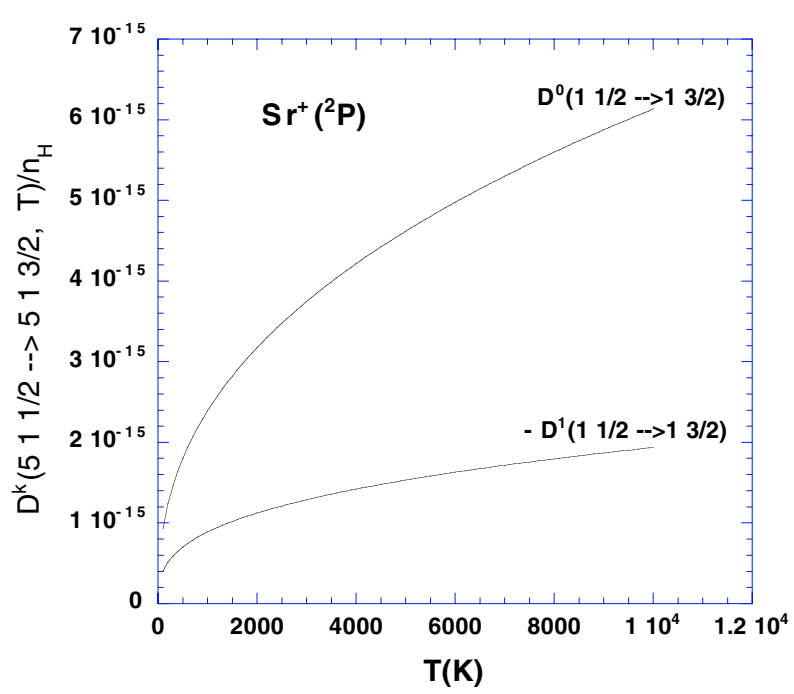

Fig. 14. Population and orientation transfer rates $(k=0$ and $k=1)$ for the SrII ion, per unit $\mathrm{H}$-atom density, as a function of temperature $T$. The rates are given in $\mathrm{rad} \mathrm{m}^{3} \mathrm{~s}^{-1}$.

\section{Conclusion}

We have adapted our semi-classical method of calculation of collisional depolarization of spectral lines of neutral atoms by atomic hydrogen to allow it to be used for singly ionised atoms. Comparison with recent quantum chemistry calculations for CaII at $T=5000 \mathrm{~K}$ indicates an error less of than $8 \%$. This is an encouraging result which supports the validity of our semiclassical approach. Using this method we should be able to calculate depolarization rates of the levels involved in transitions of heavy ionised atoms like SrII, Ti II, Ce II, Fe II, Cr II, BaII... Calculations must proceed line by line because a suitable $E_{\mathrm{p}}$ value needs to be determined for each relevant state of the given ion. We have applied our general method to calculate depolarization and polarization transfer rates for the SrII $5 p{ }^{2} \mathrm{P}$ state. These calculations should allow a more accurate theoretical interpretation of the observed linear polarization of the SrII $4078 \AA$ line.

\section{References}

Anstee, S. D., \& O’Mara, B. J. 1991, MNRAS, 253, 549

Anstee, S. D. Ph.D. Thesis, Univ. Queensland, 1992

Anstee, S. D., \& O’Mara, B. J. 1995, MNRAS, 276, 859

Barklem, P. S., \& O'Mara, B. J. 1997, MNRAS, 290, 102

Barklem, P. S., \& O'Mara, B. J. 1998, MNRAS, 296, 1057

Barklem, P. S., \& O’Mara, B. J. 1998, MNRAS, 300, 863

Barklem, P. S., \& O'Mara, B. J. 2000, MNRAS, 311, 535

Barklem, P. S. Ph.D. Thesis, Univ. Queensland, 1998

Barklem, P. S., Piskunov, N., \& O'Mara, B. J. 2000, A\&A, 142, 467

Bianda, M., Stenflo, J. O., \& Solanki, S. K. 1998, A\&A, 337, 565

Bommier, V., \& Molodij, G. 2002, A\&A, 381, 241

Dalgarno, A., \& Williams, D. A. 1962, Proc. Phys. Soc. A (London), 69,57

Derouich, M., Sahal-Bréchot, S., Barklem, P. S., \& O’Mara, B. J. 2003a, A\&A, 404, 763 (Paper I)

Derouich, M., Sahal-Bréchot, S., \& Barklem, P. S. 2003b, A\&A, 409, 369 (Paper II)

Derouich, M., Sahal-Bréchot, S., \& Barklem, P. S. 2004, A\&A, 414, 369 (Paper III)

Dittmann, O., Trujillo Bueno, J., Semel, M., \& López Ariste, A. 2001, in Advanced Solar Polarimetry: Theory, Observation, and Instrumentation, ed. M. Sigwarth, ASP Conf. Ser., 236, 125

Fano, U., \& Racah, G. 1959, Irreducible Tensorial Sets (New York: Academic Press)

Fano, U. 1963, Phys. Rev., 131, 259

Gandorfer, A. 2000, The Second Solar Spectrum: A high spectral resolution polarimetric survey of scattering polarization at the solar limb in graphical representation, vol. 1: $4625 \AA$ to $6995 \AA$ (Hochschulverlag AG an der ETH Zurich)

Gandorfer, A. 2002, The Second Solar Spectrum: A high spectral resolution polarimetric survey of scattering polarization at the solar limb in graphical representation, vol. 2: $3910 \AA$ to $4630 \AA$ (Hochschulverlag AG an der ETH Zurich)

Goodisman, J. 1973, Diatomic Interaction Potential Theory (New York: Academic Press)

Kerkeni, B. 2003, A\&A, 402, 5

Manso Sainz, R., \& Trujillo Bueno, J. 2001, in Advanced Solar Polarimetry: Theory, Observation, and Instrumentation, ed. M. Sigwarth, ASP Conf. Ser., 236, 213

Manso Sainz, R., \& Trujillo Bueno, J. 2003, Phys. Rev. Lett., 91, 11

Messiah, A. 1961, Mécanique Quantique (Paris: Dunod)

Sahal-Bréchot, S. 1977, ApJ, 213, 887

Seaton, M. J. 1958, MNRAS, 118, 504

Stenflo, J. O., Baur, T. G., \& Elmore, D. F. 1980, A\&A, 84, 60

Stenflo, J. O., Twerenbold, D., \& Harvey, J. W. 1983a, A\&A, 52, 161, NASA ADS

Stenflo, J. O., Twerenbold, D., Harvey, J. W., \& Brault, J. W. 1983b, A\&AS, 54, 505

Stenflo, J. O., \& Keller, C. U. 1997, A\&A, 321, 927

Stenflo, J. O., Keller, C. U., \& Gandorfer, A. 2000, A\&A, 355, 789

Trujillo Bueno, J., \& Collados, M., Paltou, F., \& Molodij, G. 2001, in Advanced Solar Polarimetry, Proc. 20th NSO/Sac Peak Summer Workshop, ed. M. Sigwarth, ASP Conf. Ser., 236, 141

Trujillo Bueno, J., \& Manso Sainz, R. 2001, in Magnetic Fields Across the Hertzsprung-Russell Diagram, ed. G. Mathys, S. K. Solanki, \& D. T. Wickramasinghe (San Francisco: ASP), ASP Conf. Ser., 248,83

Unsöld, A. L. 1927, Zeitschrit für Physik, 43, 574

Unsöld, A. L. 1955, Physik der Stern Atmosphären (Zweite Auflage) 